\title{
Diagnostic significance of circulating immune complexes in patients with pulmonary tuberculosis
}

\author{
V. V. RADHAKRISHNAN, A. MATHAI and P. SUNDARAM* \\ Department of Pathology, Sree Chitra Tirunal Institute for Medical Sciences and Technology, Trivandrum-0695 011. \\ and "Hospital for Tuberculosis and Chest Diseases, Trivandrum, India
}

\begin{abstract}
Summary. A polyethylene glycol (PEG) precipitation method was used to examine sera of patients with active pulmonary tuberculosis (PT), leprosy and non-tuberculous pulmonary diseases and of healthy control subjects for immune complexes (ICs). Mycobacterium tuberculosis antigen 5 was detected in the ICs in $80 \%$ of patients with PT by the indirect (sandwich) enzyme-linked immunosorbent assay (ELISA). Detection of mycobacterial antigen in ICs has diagnostic potential as an adjunct in the laboratory diagnosis of PT, particularly when repeated bacteriological investigations for $M$. tuberculosis in clinical specimens are negative. Levels of ICs tend to decrease with the duration of anti-tuberculosis chemotherapy and their detection can also be used to assess the clinical response to therapy in patients with PT.
\end{abstract}

\section{Introduction}

Early diagnosis of patients with active pulmonary tuberculosis (PT) is essential for effective initiation of appropriate chemotherapy. Diagnosis of this disease depends upon the specific demonstration of $M y c o b a c-$ terium tuberculosis in clinical specimens. Bacteriological culture is both cumbersome and time consuming but detection of acid-fast bacilli in sputum smears is less sensitive. ${ }^{1}$ For several years, enzyme-linked immunosorbent assays (ELISA) have been used to aid the diagnosis of tuberculosis. ${ }^{2-4}$ In these studies, $M$. tuberculosis antigen 5 has been used because this antigen is considered to be more specific than other mycobacterial antigens. Daniel and Anderson ${ }^{5}$ isolated this antigen from the unheated cell-free culture filtrate of $M$. tuberculosis strain $\mathrm{H}_{37} \mathrm{Ra}$ by means of immunoabsorbent affinity chromatography and called it $M$. tuberculosis antigen 5 because it identified with this number in the nomenclature of mycobacterial antigens. ${ }^{6}$ By immuno-electrophoresis, this antigen gave a single precipitin arc with polyvalent rabbit antiserum to $M$. tuberculosis. ${ }^{7}$ Physicochemical studies have shown that antigen 5 is composed of amino acids rich in aspartic acid, suggesting that it is derived from the cytoplasm of $M$. tuberculosis. It does not contain muramic or diaminopimelic acids, which further suggests that antigen 5 is not derived from the cell wall of $M$. tuberculosis. Antigen 5 is stable at $70^{\circ} \mathrm{C}$ and, by sodium docecylsulphate-polyacrylamide gel electrophoresis (SDS-PAGE), it has been characterised as a single band of $35 \mathrm{Kda} .{ }^{5}$

Received 10 Oct. 1990; revised version accepted 10 May 1991.
The present study was undertaken (i) to isolate and characterise immune complexes (ICs) in sera of patients with PT, (ii) to evaluate the diagnostic relevance of detection of $M$. tuberculosis antigen 5 in the ICs by a sandwich ELISA and (iii) to correlate IC levels with clinical recovery during and after chemotherapy in patients with PT.

\section{Materials and methods}

\section{Patients and serum samples}

Sera from four groups of patients were examined. (A) Sera from 50 patients admitted to the Hospital for Tuberculosis and Chest Disease, Trivandrum, with a clinical diagnosis of PT made by the attending consultant physician and supported by radiological investigations. $M$. tuberculosis was demonstrated in sputum specimens from 15 patients. In the remaining 35 patients, repeated sputum examinations and cultures were negative for $M$. tuberculosis and atypical mycobacteria. At the time of completion of this study, all the patients had received continuous anti-tuberculosis chemotherapy for 1-6 months. Sera were collected also from 10 asymptomatic patients with PT who had received continuous therapy for 6-12 months. (B) Sera from 40 patients with non-tuberculous pulmonary diseases (bronchogenic carcinoma 16, chronic bronchitis 18 , pneumococcal pneumonia 6) were collected. (C) Sera from 50 healthy voluntary blood donors attending the Blood Transfusion Services. (D) Sera from 10 patients with lepromatous and tuberculoid leprosy. None of the patients in clinical groups B, C and $D$ showed either clinical or radiological evidence 
of active PT. These patients were selected as controls for this study. Sera from tuberculous and control groups were coded and preserved at $-70^{\circ} \mathrm{C}$ in sterile storage vials.

\section{Antigen}

M. tuberculosis antigen 5 was isolated from the unheated cell-free culture filtrates of $M$. tuberculosis strain $\mathrm{H}_{37} \mathrm{Ra}$ by immuno-absorbent affinity chromatography ${ }^{5}$ with reference goat antiserum to $M$. tuberculosis antigen 5 (kindly supplied by Dr T. M. Daniel, Cleveland, OH, USA).

\section{Antiserum to $M$. tuberculosis antigen 5}

Adult male rabbits were used to raise the antiserum. A water-in-oil emulsion containing $1 \mathrm{ml}(100 \mu \mathrm{g})$ of antigen 5 and $1 \mathrm{ml}$ of Bordetella pertussis vaccine (Pasteur Institute, Kasuli, India) were mixed thoroughly in $1 \mathrm{ml}$ of incomplete Freund's adjuvant. The material was injected intramuscularly and subcutaneously at multiple sites. The same procedure was repeated at 14,21, 35 and 42 days after the primary immunisation. In Ouchterlony immunodiffusion plates, the antiserum demonstrated a single precipitin arc against both $M$. tuberculosis antigen 5 and a culture filtrate of $M$. tuberculosis. The antiserum was passed through a protein A-sepharose column ${ }^{8}$ and the immunoglobulin $\mathrm{G}(\mathrm{IgG})$ fraction was recovered. This IgG fraction was dialysed extensively and concentrated with an Amicon ultrafiltration unit (Amicon $\mathrm{GmbH}$, Written, Germany). The protein content was estimated by Lowry's method ${ }^{9}$ and the IgG fraction was preserved at $-70^{\circ} \mathrm{C}$ in sterile storage vials.

\section{Precipitation of ICs}

ICs in sera were precipitated with PEG 6000 (Sigma). ${ }^{10}$ Briefly, $0.8 \mathrm{ml}$ of the serum sample was mixed with an equal amount of $0.2 \mathrm{M}$ EDTA and centrifuged at $700 \mathrm{~g}$ for $20 \mathrm{~min}$. To the supernate, $0.4 \mathrm{ml}$ of PEG $12 \% \mathrm{w} / \mathrm{v}$ in $0.1 \mathrm{M}$ sodium borate buffer, $\mathrm{pH} 8.4$, was added and the mixture was incubated for $12 \mathrm{~h}$ at $4^{\circ} \mathrm{C}$. ICs were collected by centrifugation at $5000 \mathrm{~g}$ for $30 \mathrm{~min}$. The pellets were washed twice with PEG washing solution (PEG $2.5 \% \mathrm{w} / \mathrm{v}, 0 \cdot 1 \mathrm{M} \mathrm{NaCl}$, $0.025 \mathrm{M}$ sodium borate, $0.08 \mathrm{M}$ EDTA and $0.1 \mathrm{M}$ Tris, $\mathrm{pH} 7 \cdot 1$ ) and resuspended in $0 \cdot 15 \mathrm{M}$ phosphate-buffered saline $\mathrm{pH} 7.4$ (PBS). Usually, the PEG precipitate from $0.8 \mathrm{ml}$ of serum was resuspended in $150 \mu \mathrm{l}$ of PBS. The presence of IgG and complement (C3 component) in PEG precipitates were determined by the single radial immunodiffusion technique (HoechstBehring) with quantitation according to the protocol supplied by the manufacturer; the technique detected $\mathrm{IgG}$ and $\mathrm{C} 3$ at levels as low as $20 \mathrm{mg} / 100 \mathrm{~g}$ and $5 \mathrm{mg} /$ $100 \mathrm{~g}$, respectively.

\section{Detection of mycobacterial antibody in PEG precipitates}

A non-competitive ELISA ${ }^{11}$ was standardised in polyvinyl chloride microtitration ELISA plates (Dynatech Inc, Alexandria, VA, USA). The reaction temperature and assay volume were $21^{\circ} \mathrm{C}$ and $100 \mu \mathrm{l}$, respectively. The PEG precipitates of test sera were serially diluted (1 in 10-1 in 80) in 0.15 M PBS before use in the assay. Briefly, the microtitration plates were coated with $M$. tuberculosis antigen $5(2 \mu \mathrm{g} /$ well in carbonate buffer, $\mathrm{pH} 9.6$ and the amount of antibody to antigen 5 in PEG precipitates was determined by the addition of anti-human IgG-alkaline phosphatase conjugate (Sigma) diluted 1 in 1000. P-Nitrophenyl phosphate $1 \mathrm{mg} / \mathrm{ml}$ in $1 \mathrm{M}$ diethanolamine buffer, $\mathrm{pH} 9 \cdot 8$, was used as the substrate. The colour reaction was stopped after $30 \mathrm{~min}$ with $3 \mathrm{~N} \mathrm{NaOH}$. The absorbances in all samples were measured at $405 \mathrm{~nm}$ with an automated ELISA reader (Titertek-multiscan; Flow laboratories, USA). The assay on PEG precipitates of the tuberculous and the control groups was repeated on two different occasions and the interassay variation (coefficient of variation) was in the range $5-8 \%$. The "cut-off" titre for the PEG precipitate was determined to be 80 , on the basis that it gave the best discrimination between tuberculous patients and controls. At this "cut-off" titre, none of the PEG precipitates in the control group gave a positive colour reaction. Test sera with an ELISA value, at a dilution of 80 , that was above the control mean $+2 \mathrm{SD}$, were considered to be positive.

\section{Detection of $M$. tuberculosis antigen 5 in the PEG precipitates}

The mycobacterial antigen was dissociated from the PEG precipitates with $4 \mathrm{M}$ urea and the ELISA test was done on the dissociated samples. Inconsistent results were obtained with dissociated PEG precipitates and, as this could have been attributable to the presence of urea in the samples, the ELISA test was performed only on undissociated PEG precipitates for all patients. A sandwich method was standardised ${ }^{12}$ in microtitration ELISA plates. The assay volume, reaction temperature and incubation time were $100 \mu 1$, $37^{\circ} \mathrm{C}$ and $3 \mathrm{~h}$ respectively. The plates were intermittently washed with 0.15 M PBS in Tween-20 (PBS-T) during the procedure. Briefly, the wells in microtitration plates were coated with rabbit $\operatorname{IgG}$ to $M$. tuberculosis antigen $5(5 \mu \mathrm{g}$ per well in carbonate buffer, $\mathrm{pH}$ 9.6) and the plates were quenched with bovine serum albumin $3 \% \mathrm{w} / \mathrm{v}$. The PEG precipitates, diluted 1 in 80 , from tuberculous and control groups were added in duplicate. The plates were incubated with anti-human IgG-alkaline phosphatase conjugate diluted 1 in 1000 . The enzyme activity was assayed by the addition of nitrophenyl phosphate. The absorbance in every sample was recorded at $405 \mathrm{~nm}$. The mean and SD of the absorbance at a dilution of 1 in 
80 were calculated for the patients in the control group. A test was considered to be positive if the absorbance in a test sample was $>2$ SD above the mean value for the control. The assay was repeated on three occasions and with coded samples, to eliminate observer bias; the inter-assay variation (co-efficient of variation) was $6-8 \%$.

\section{Results}

The table records the incidence of ICs detected (antigen 5, IgG antibody and C3 component of complement) in the PEG precipitates of sera from patients with PT, leprosy, non-tuberculous pulmonary diseases and healthy controls. The ICs were detected, with different incidences, in the two PT and the leprosy groups, but not in the non-tuberculous or healthy control groups. Fig. 1 shows the ELISA data for antigen 5 in the various clinical categories. In the 35 culture-negative patients with PT, absorbances (OD) in the ELISA for antigen 5 were in the range $0.28-0.69$ (mean 0.57 SD 0.15). The mean absorbance in 15 culture-positive patients with PT was 0.592 SD $0 \cdot 11$. In patients with non-tuberculous pulmonary diseases and healthy controls, the mean absorbances in the ELISA tests were 0.249 SD 0.13 and 0.202 SD 0.09 respectively. A test was considered to be positive for the presence of antigen 5 if the absorbance was $>0.509$ (the mean absorbance + $2 \mathrm{SD}$ in the non-tuberculous group). By this criterion, with sera from 25 out of 35 culture-negative patients with PT and all the 15 culture-positive patients with PT, results were positive for antigen 5 in the ICs. By the same criterion, sera from two of 10 patients with leprosy were considered to be positive for the presence of antigen 5 in the ICs, but none of the sera from 40 non-PT and 50 healthy control patients gave a positive result.

Fig. 2 shows the ELISA data for anti-antigen $5 \mathrm{IgG}$ antibody in ICs from patients with PT and leprosy and from non-tuberculous subjects. Anti-mycobacterial antibodies were present in ICs from all the 15

Table. Incidence of ICs in PEG precipitates of sera from patients in various clinical categories

\begin{tabular}{ll}
\hline \multicolumn{1}{c}{ Patient groups (n) } & $\begin{array}{c}\text { Percentage of patients with } \\
\text { detectable ICs }\end{array}$ \\
\hline $\begin{array}{l}\text { Culture-positive, with pulmonary } \\
\text { tuberculosis (15) }\end{array}$ & $100(15)^{*}$ \\
$\begin{array}{l}\text { Culture-negative, with pulmonary } \\
\text { tuberculosis (35) }\end{array}$ & $71(25)$ \\
$\begin{array}{l}\text { Leprosy (10) } \\
\text { Non-tuberculous pulmonary } \\
\text { diseases (40) }\end{array}$ & $40(4)$ \\
Health controls (50) & 0 \\
\end{tabular}

* Figures in parentheses are number of samples that gave positive results.

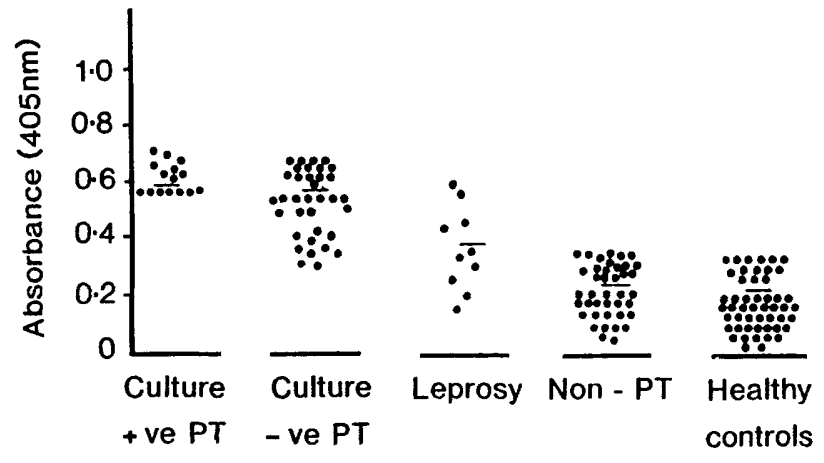

Fig. 1. Detection of $M$. tuberculosis antigen 5 by indirect ELISA in the ICs from patients in various clinical categories. Each dot represents the absorbance value recorded for an individual patient. The horizontal lines represent the mean value for each group.

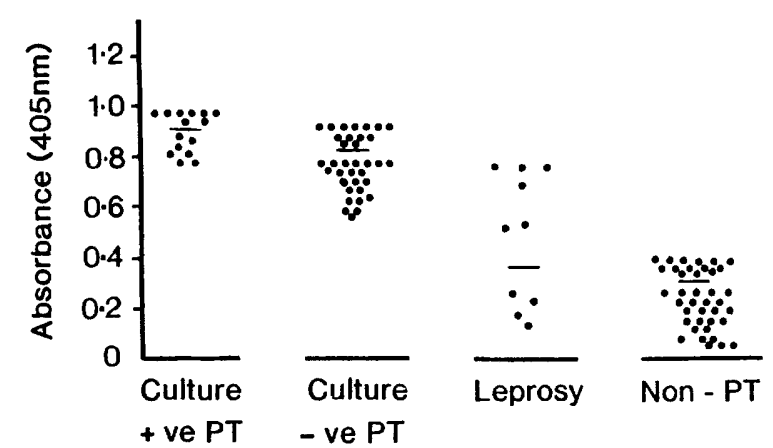

Fig. 2. Detection of IgG antibody to $M$. tuberculosis antigen 5 by ELISA in ICs of tuberculous, leprosy and non-tuberculous patients. Each dot represents the absorbance value recorded for an individual patient. The horizontal line represents the mean value for each group.

culture-positive patients with PT and absorbances in the ELISA were in the range 0.79-0.97 (mean $0.86 \mathrm{SD} 0 \cdot 24$ ). In sera from 35 culture-negative patients with PT, the absorbances in the ELISA varied between 0.52 and 0.93 (mean 0.81 SD 0.22 ). The mean absorbance in samples from patients with nontuberculous pulmonary diseases was $0.31 \mathrm{SD} 0 \cdot 21$. Therefore, a test was considered to be positive when the absorbance value was $>0.72$ (mean absorbance $+2 \mathrm{SD}$ in non-tuberculous subjects). By this criterion, sera from 25 out of 35 culture-negative patients with PT had anti-mycobacterial antibody in the ICs. The absorbance values for four of the patients with leprosy were $>0.72$ and were considered to be positive for anti-mycobacterial antibody in their ICs. Two of these had antigen 5 as a component of the ICs. Thus, both antigen 5 and anti-mycobacterial (antigen 5) antibody could be detected in ICs of $80 \%$ of patients with clinically diagnosed PT (including culture-positive and culture-negative cases).

For both types of ELISA test, the differences in the mean values from culture-positive and culture-negative patients were not statistically significant $(p>$ 0.05). PEG precipitates from patients with nontuberculous pulmonary diseases and healthy controls did not contain detectable amounts of either antigen 5 or the anti-mycobacterial antibody. PEG precipitates in 10 patients with PT who had received continuous 
chemotherapy for more than 6 months showed neither antigen 5 nor IgG antibody in their ICs, whereas in patients who had received chemotherapy for less than 6 months, both factors were present in the ICs.

\section{Discussion}

The presence of ICs in patients with active pulmonary tuberculosis ${ }^{13-16}$ and their diagnostic significances ${ }^{12,17}$ have been emphasised in published reports. Bhattacharaya $e a^{17}$ have reported an ELISA for the determination of ICs in tuberculous patients. They have stated that the antibody present in ICs was predominantly $\mathrm{IgG}$, and was specific for $M$. tuberculosis antigen. In their study, although an immuno-dot assay was used to detect antigen in ICs, information about defined antigenic components of $M$. tuberculosis in the ICs was not presented. Moreover, only a small number of tuberculous subjects was studied and patients with non-tuberculous pulmonary diseases were not included among the control group. Mehta and $\mathrm{K}_{\text {huller }}{ }^{12}$ have developed an ELISA test to detect phosphatidylinositomannoside, a glycolipid mycobacterial antigen in ICs of patients with PT. Their assay had a sensitivity of $88 \%$ and $66 \%$, respectively, in sputum-smear-positive and smear-negative patients with PT. However, in that study, patients with nontuberculous pulmonary diseases were not included. Because the specificity of ELISA should be evaluated in non-tuberculous subjects, patients with non-tuberculous pulmonary diseases were selected as controls in the present study.

\section{References}

1. Yáñez MA, Coppola MP, Russo DA, Delaha E, Chaparas SD, Yeager $\mathrm{H}$. Determination of mycobacterial antigens in sputum by enzyme immunoassay. J Clin Microbiol 1986; 23: 822-825.

2. Balestrino EA, Daniel TM, De Latini MDS, Latini OA, Ma Y, Scocozza JB. Serodiagnosis of pulmonary tuberculosis in Argentina by enzyme-linked immunosorbent assay (ELISA) for IgG antibody to Mycobacterium tuberculosis antigen 5 and tuberculin purified positive derivative. Bull WHO 1984; 62: 755-761

3. Ma Y, Wang Y-M, Daniel TM. Enzyme-linked immunosorbent assay using Mycobacterium tuberculosis antigen 5 for the diagnosis of pulmonary tuberculosis in China. Am Rev Respir Dis 1986; 134: 1273-1275.

4. Daniel TM, Debanne SM, Van der Kuyp F. Enzyme-linked immunosorbent assay using Mycobacterium tuberculosis antigen 5 and PPD for serodiagnosis of tuberculosis. Chest 1985; 88: 388-392.

5. Daniel TM, Anderson PA. The isolation by immunoabsorbent affinity chomatography and physicochemical characterization of Mycobacterium tuberculosis antigen 5. Am Rev Respir Dis 1978; 117: 533-539.

6. Janicki BW, Chaparas SD, Daniel TM, Kubica GP, Wright GL, Yee GS. A reference system for antigens of $M y c o b a c-$ terium tuberculosis. Am Rev Respir Dis 1971; 104: 602-604.

7. Radhakrishnan VV, Mathai A, Rao SB, Sehgal S. Immunoelectrophoresis of mycobacterial antigens. Indian J Exp Biol $1990 ; 28: 812-815$.

8. Golding JW. Use of staphylococcal protein A as an immunological reagent. J Immunol Methods $1978 ; 20: 241-253$.

9. Lowry OH, Rosebrough NJ, Farr AL, Randall RJ. Protein measurement with the Folin phenol reagent. $J$ Biol Chem $1951 ; 193: 265-275$.
Daniel et al. ${ }^{18}$ found that antigen 5 was present only in six strains of $M$. tuberculosis and four strains of $M$. bovis examined and that it was not present in 30 strains of 12 other mycobacterial species. Therefore, it is unlikely that sera of patients with atypical mycobacterial pulmonary diseases would react with antigen 5 in the ELISA test and the possibility of atypical mycobacterial diseases can be excluded in our patients with positive results. However, the antibodies present in PEG precipitates in four out of 10 patients with leprosy did react with antigen 5 in our ELISA test; therefore, we consider that the presence of antigen 5 is not restricted to $M$. tuberculosis.

In conclusion, two relevant observations in this study need to be emphasised: (a) $M$. tuberculosis antigen 5 in ICs can be demonstrated in $80 \%$ of patients with PT by an indirect (sandwich) ELISA test and this assay has diagnostic potential in those patients for whom standard bacteriological tests for $M$. tuberculosis give negative results; (b) ICs are detectable during the active state of the disease and tend to decrease with the duration of chemotherapy. Therefore this criterion can also be applied as one of the parameters for assessing the clinical response to anti-tuberculosis chemotherapy in patients with PT. The presence of ICs in sera from such patients would suggest that the disease was still in an active stage.

We thank the Director, Sree Chitra Tirunal Institute for Medica Sciences and Technology, Trivandrum-695 011, for his kind permission to publish this article. We are indebted to Dr T.M Daniel, Department of Medicine, Case Western Reserve University School of Medicine, Cleveland, OH 44106, USA, for the kind supply of reference $M$. tuberculosis antigen 5

10. Paganelli R, Levinsky RJ, Atherton DJ. Detection of specific antigen within circulating immune complexes: validation of the assay and its application to food antigen-antibody complexes formed in healthy and food-allergic subjects. Clin Exp Immunol 1981 ; 46: 44-53.

11. Engvall E, Perlmann P. Enzyme-linked immunosorbent assay, ELISA, III. Quantitation of specific antibodies by enzymelabeled anti-immunoglobulin in antigen-coated tubes. $J$ Immunol 1972; 109: 129-135.

12. Mehta PK, Khuller GK. Comparative evaluation of the diagnostic significance of circulating immune complexes and antibodies to phosphatidylinosito-mannosides in pulmonary tuberculosis by enzyme-linked immunosorbent assay. Med Microbiol 1989; 178: 229-233.

13. Carr RI, Chakraborty AK, Brunda MJ et al. Immune complexes and antibodies to BCG in sera from patients with mycobacterial infections. Clin Exp Immunol 1980; 39: 562 569.

14. Brostoff $\mathbf{J}$, Lenzini $\mathbf{L}$, Rottoli $\mathbf{P}$, Rottolo L. Immune complexes in the spectrum of tuberculosis. Tubercle 1981; 62: 169173.

15. Johnson N McL, McNicol MW, Burton-Kee EJ, Mowbray JF. Circulating immune complexes in tuberculosis. Thorax $1981 ; 36: 610-617$

16. May JJ, Katilus J, Hensen PM, Dreisin RB. The purification and identification of circulating immune complexes in tuberculosis. Am Rev Respir Dis 1983; 128 : 920-925.

17. Bhattacharaya A, Ranadive SN, Kale $M$, Bhattacharaya S. Antibody-based enzyme-linked immunosorbent assay for determination of immune complexes in clinical tuberculosis. Am Rev Respir Dis 1986; 134 : 205-209.

18. Daniel TM, Ellner JJ, Todd LS et al. Immunobiology and species distribution of Mycobacterium tuberculosis antigen 5. Infect Immun 1979; 24:77-82. 